\title{
Quantum Optics with Quantum Dots in Photonic Nanowires
}

\author{
Gérard, J. M. ; Claudon, J. ; Bleuse, J.; Munsch, M. ; Gregersen, Niels; Lalanne, P .
}

Published in:

CLEO Technical Digest

Publication date:

2012

Document Version

Publisher's PDF, also known as Version of record

Link back to DTU Orbit

Citation (APA):

Gérard, J. M., Claudon, J., Bleuse, J., Munsch, M., Gregersen, N., \& Lalanne, P. . (2012). Quantum Optics with Quantum Dots in Photonic Nanowires. In CLEO Technical Digest (pp. CTh3M.1). Optical Society of America.

\section{General rights}

Copyright and moral rights for the publications made accessible in the public portal are retained by the authors and/or other copyright owners and it is a condition of accessing publications that users recognise and abide by the legal requirements associated with these rights.

- Users may download and print one copy of any publication from the public portal for the purpose of private study or research.

- You may not further distribute the material or use it for any profit-making activity or commercial gain

- You may freely distribute the URL identifying the publication in the public portal

If you believe that this document breaches copyright please contact us providing details, and we will remove access to the work immediately and investigate your claim. 


\title{
Quantum Optics with Quantum Dots in Photonic Nanowires
}

\author{
J.M. Gérard ${ }^{1 *}$, J. Claudon ${ }^{1}$, J Bleuse ${ }^{1}$, M. Munsch ${ }^{1}$, N. Gregersen ${ }^{2}$ and P Lalanne ${ }^{3}$ \\ ${ }^{1}$ CEA-CNRS joint group 'NanoPhysique et SemiConducteurs', CEA, INAC, SP2M, 38054 Grenoble, France \\ ${ }^{2}$ DTU Fotonik, Technical University of Denmark, DK-2800 Kongens Lyngby, Denmark \\ ${ }^{3}$ Laboratoire Charles Fabry de l'Institut d'Optique 91127 Palaiseau, France
}

\begin{abstract}
We review recent experimental and theoretical results, which highlight the strong interest of the photonic wire geometry for solid-state quantum optics and quantum optoelectronic devices.

OCIS codes : (270.5580), (350.4238), (250.5590)
\end{abstract}

\section{Introduction}

Over the last 20 years, major efforts have been devoted to the tailoring of the optical properties of semiconductor emitters using optical microcavities and photonic crystals. Photonic wires, initially introduced in the context of microlasers [1], have recently been used by our group to control the spontaneous emission of self-assembled InAs quantum dots (QDs) and to build highly efficient single-mode single photon sources. We use in these experiments cylindrical GaAs photonic nanowires, defined using a top-down approach based on electron-beam lithography and reactive-ion etching (see fig.1)

\section{QD spontaneous emission control in photonic wires}

For an optimum diameter $\mathrm{d} / \lambda \sim 0.2$, such structures provide a strong lateral confinement of the guided mode, while the amplitude of non-guided modes is drastically damped within the wire due to a dielectric screening effect [2]. As a result, theoretical calculations show that the fraction of the spontaneous emission (SE) that is coupled to the guided mode $\mathrm{HE}_{11}$ reaches $\beta=95 \%$ for an emitter on-axis, provided the polarization of its dipole is orthogonal to the wire axis [3]. Unlike optical microcavities, which exploit the SE enhancement into the cavity mode (Purcell effect), photonic wires thus rely on the inhibition of the SE into "useless" modes for providing this "nearly" single-mode behavior of the SE.

The study of QDs embedded in photonic wires by time-resolved PL highlights this crucial inhibition effect at work. Very long lifetimes (up to x 17 v.s. QDs in bulk GaAs) are observed for those QDs that are weakly coupled to the guided mode [2]. This is the case for QDs located close to the wire edge, or for QDs in very narrow wires $(\mathrm{d} / \lambda<0.15)$, for which the guided mode is mostly located outside the wire.
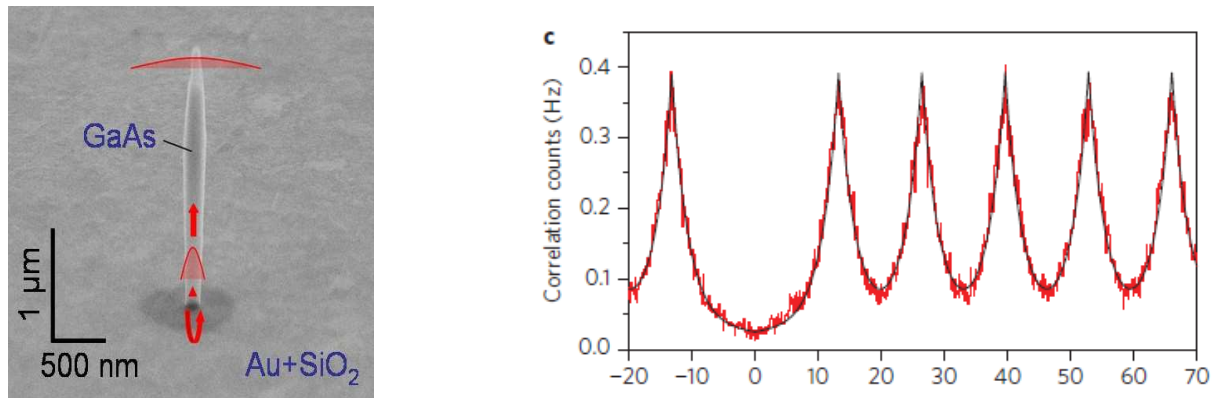

Figure 1: (left) View by scanning electron microscopy of a tapered GaAs photonic wire on top of an hybrid $\mathrm{Au} / \mathrm{SiO} 2$ mirror. (right) Photon correlation histogram obtained for the exciton line of a QD in a photonic wire at saturation, under pulsed optical excitation. The fit to theory reveals the absence of multi-photon pulses $\left(\mathrm{g}^{(2)}(0)<0.008\right)$. From [7].

Photonic wires with an axial symmetry support two polarization-degenerate guided modes. In view of applications, it is often desirable to control also the polarization of the spontaneous emission of embedded emitters. In micropillar resonators, a breaking of the axial symmetry can be used to lift the degeneracy of the fundamental cavity mode, and couple the emitter to a single and polarized cavity mode [4]. In a similar spirit, we have studied photonic nanowires with an elliptical cross-section. For dimensions of the ellipse around $\lambda / n$ (long axis) and $\lambda / 2 n$ (short axis), the two guided modes exhibit drastically different behaviours. The first mode, with a linear polarization 
oriented along the ellipse major axis, is tightly confined inside the wire, while the second mode is mostly located outside the photonic wire. As a result, one expects unpolarized semiconductor emitters (such as quantum well or quantum dot excitons) to display a truly single-mode, linearly polarized spontaneous emission, when embedded in such photonic wires. Furthermore, this single mode behaviour is maintained over a broad wavelength range, since no resonant effect enters into play. Experiments on elliptical GaAs photonic wires with embedded InAs quantum dots fully confirms these predictions [4]. In particular, the fraction of collected photons with the desired linear polarization reaches as much as $95 \%$ for the best QDs, located close to the wire axis.

\section{Application to single mode single-photon sources}

A highly efficient single photon source (SPS) based on a QD in a photonic wire has recently been developed [3]. In order to ensure a good collection of the single photons, the far field radiation diagram of the wire is carefully tailored using a broadband $\mathrm{SiO}_{2}-\mathrm{Au}$ modal mirror at the bottom side [5] and a smooth tapering of the wire tip [6]. Combining these building blocks, one predicts a record high SPS efficiency (>90\%) over a 70 nm-broad spectral range for standard collection optics (NA 0.75) [2]. The optical characterization performed using microPL and photon correlation experiments fully confirm the theoretical predictions: a record high efficiency (72\%) is obtained [7], to be compared to $40 \%$ at most for the best published results for SPS based on cavity effects. Furthermore, a very pure single photon emission $\left(g^{(2)}(0)<0.01\right)$ is observed for all pumping powers, up to the saturation of the emission of the QD exciton [7]. This is a crucial asset $v s$ cavity-based QD-SPS, whose $\mathrm{g}^{(2)}(0)$ is plagued by "cavity feeding" [8] effects under strong excitation conditions $[9,10]$.

Quite remarkably, these photonic wires, with their integrated mirrors and tapered tips, provide a broadband control of the SE of embedded emitters. They thus offer appealing novel opportunities for the development of SPS based on spectrally broad emitters (such as F centers in diamond), wavelength tunable sources or efficient sources of entangled photon pairs. Finally, novel designs based on inverted tapers will also enable the efficient operation of these quantum optoelectronic devices under electrical pumping [11].

The authors gratefully acknowledge support from the French ANR projects "CAFÉ" and "WIFO", as well as N.S. Malik, E. Dupuy, P. Jaffrennou, M. Creasey, I. Maksymov, J.P. Hugonin, Y. Chen and J. Moerk for their contributions and support to this work.

\section{References}

[1] J.P. Zhang et al, "Photonic-wire lasers", Phys. Rev. Lett. 75, 2678 (1995)

[2] J.Bleuse, J. Claudon, M. Creasey, N.S. Malik, J.M. Gérard, I. Maksymov, J.P. Hugonin, P Lalanne, "Inhibition, enhancement and control of spontaneous emission in photonic nanowires”, Phys. Rev. Lett. 106, 103601 (2011)

[3] I. Friedler, C. Sauvan, J.P. Hugonin, P. Lalanne, J. Claudon and J.M. Gérard, "Solid-state single photon sources : the nanowire antenna", Optics Express 17, 2095-2110 (2009)

[4] M. Munsch, J. Claudon, J. Bleuse, N.S. Malik, E. Dupuy, J.M. Gérard, Y. Chen, N. Gregersen, J. Moerk, to appear in Phys. Rev. Lett. $(02 / 2012)$

[5] J. Claudon, J. Bleuse, M. Bazin, N.S. Malik, P. Jaffrennou, N. Gregersen, C. Sauvan, P. Lalanne and J.M. Gérard, "A highly efficient singlephoton source based on a quantum dot in a photonic wire", Nature Photon. 4, 174 (2010)

[6] I. Friedler, P. Lalanne, J.P. Hugonin, J. Claudon, J.M. Gérard, A. Beveratos, I. Robert-Philip, "Efficient photonic mirrors for semiconductor nanowires", Opt. Lett. 33, 2635 (2008)

[7] N. Gregersen, T.R. Nielsen, J. Claudon, J.M. Gérard and J. Moerk, "Controlling the emission profile of a nanowire with a conical taper", Opt. Lett. 33, 1693 (2008)

[8] A. Auffèves, B. Besga, J.M. Gérard and J.P. Poizat, "Spontaneous emission spectrum of a two-level atom in a very high Q cavity", Phys. Rev. A $77,063833(2008)$

[9] E. Moreau et al, "A single-mode solid-state source of single photons based on isolated quantum dots in a Micropillar", Physica E 13, 418-422 (2002).

[10] S. Strauf et al, "High-frequency single-photon source with polarization control", Nat. Photonics 1, 704 - 708(2007).

[11] N Gregersen T.R. Nielsen, J Mork, J Claudon, J.M. Gérard, "High-efficiency electrically-pumped nanowire single-photon sources", Opt. Exp. 18, 21204(2010)

\footnotetext{
* jean-michel.gerard@cea.fr
} 